\title{
Rare anastomosis between the ascending pharyngeal and vertebral arteries via the hypoglossal canal: A cadaveric case report
}

\author{
Stephen J. Bordes ${ }^{1}$, Sina Zarrintan ${ }^{2}$, Joe Iwanaga ${ }^{3,4,10}$, Marios Loukas ${ }^{5}$, Aaron S. Dumont ${ }^{3}$, \\ R. Shane Tubbs $3,4,5,6,7,8,9$ \\ ${ }^{1}$ Department of Surgery, Louisiana State University, New Orleans, LA, USA, ${ }^{2}$ Division of Vascular and Endovascular Surgery, Department of \\ Surgery, Tabriz University of Medical Sciences, Tabriz, Iran, ${ }^{3}$ Department of Neurosurgery, Tulane University School of Medicine, New Orleans, \\ LA, ${ }^{4}$ Department of Neurology, Tulane University School of Medicine, New Orleans, LA, USA, ${ }^{5}$ Department of Anatomical Sciences, St. George's \\ University, St. George's, Grenada, ${ }^{6}$ Department of Surgery, Tulane University School of Medicine, New Orleans, LA, ${ }^{7}$ Department of Structural and \\ Cellular Biology, Tulane University School of Medicine, New Orleans, LA, ${ }^{8}$ Department of Neurosurgery, Ochsner Neuroscience Institute, Ochsner \\ Health System, New Orleans, LA, USA, ${ }^{9}$ Queensland University, Brisbane, Australia, ${ }^{10}$ Division of Gross and Clinical Anatomy, Department of \\ Anatomy, Kurume University School of Medicine, Kurume, Fukuoka, Japan
}

Abstract: We present a rare case of external carotid artery-vertebral artery anastomosis via the ascending pharyngeal artery, diagnosed upon cadaveric dissection. The ascending pharyngeal artery gave rise to a branch to the hypoglossal canal, which is a variation of a true persistent fetal hypoglossal artery. Knowledge of persistent carotid-vertebrobasilar anastomoses is important as these fetal vessels can contribute significantly to the posterior cerebral circulation. Only 10 cases of external carotid artery-vertebrobasilar artery anastomoses have been reported to our knowledge, and our case presents the first cadaveric dissection of this rare variation.

Key words: Ascending pharyngeal artery, Persistent fetal hypoglossal artery, Hypoglossal artery, Hypoglossal canal, Vertebral artery

Received May 7, 2021; Accepted June 9, 2021

\section{Introduction}

Persistent fetal, or primitive, anastomoses between the carotid and vertebrobasilar systems are known variations resulting from a failure of regression during embryogenesis. Fetal trigeminal arteries are most likely to persist followed by hypoglossal, otic, and proatlantal intersegmental arteries (Fig. 1) $[1,2]$. These variations, which typically connect the internal carotid artery to the vertebral artery, are estimated

\section{Corresponding author:}

Joe Iwanaga (i)

Department of Neurosurgery, Tulane Center for Clinical Neurosciences, Tulane University School of Medicine, New Orleans, LA 70112, USA E-mail: iwanagajoeca@gmail.com

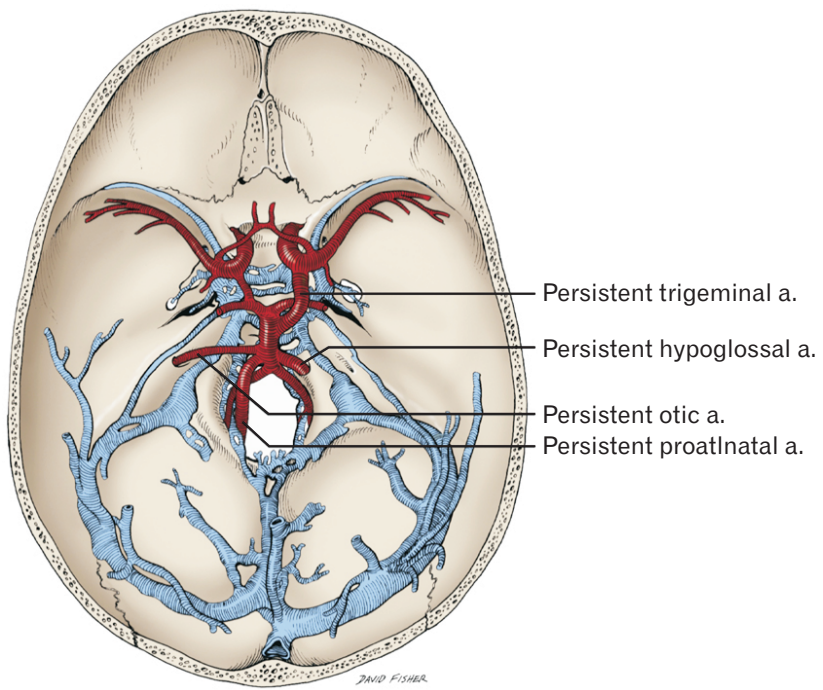

Fig. 1. The four persistent carotid-vertebrobasilar anastomoses.

\section{Copyright (C) 2021. Anatomy \& Cell Biology}

This is an Open Access article distributed under the terms of the Creative Commons Attribution Non-Commercial License (http://creativecommons.org/licenses/by-nc/4.0/) which permits unrestricted non-commercial use, distribution, and reproduction in any medium, provided the original work is properly cited. 
to occur in $0.027 \%$ to $0.26 \%$ of the population [1-4]. We present a rarer variation. An anastomosis was identified between the external carotid and vertebral arteries via a hypoglossal branch of the ascending pharyngeal artery. Only 10 cases of this uncommon variation can be found in the literature [4]. To our knowledge, our case is the first cadaveric report. All other reported cases have identified this variation using magnetic resonance or computed tomography angiography. Knowledge of these persistent fetal anastomoses is crucial as they can contribute significant blood flow to the vertebrobasilar system and posterior cerebral circulation.

\section{Case Report}

During a cadaveric head and neck dissection, the left ascending pharyngeal artery was found to give off a branch to the hypoglossal canal (Fig. 2). The specimen was a 79-yearold at death male with no signs of previous trauma or pathology to the area dissected. This hypoglossal arterial branch was followed cranially and found to join the ipsilateral vertebral artery at the level at which it gave off the posterior inferior cerebellar artery (Fig. 3). The hypoglossal artery was $1.28 \mathrm{~mm}$ in diameter and $38 \mathrm{~mm}$ long. The hypoglossal canal was not enlarged on this side. Thus, this variant anastomosis connected the external carotid artery with the vertebrobasilar system (Fig. 4). There was no similar finding on the contra-

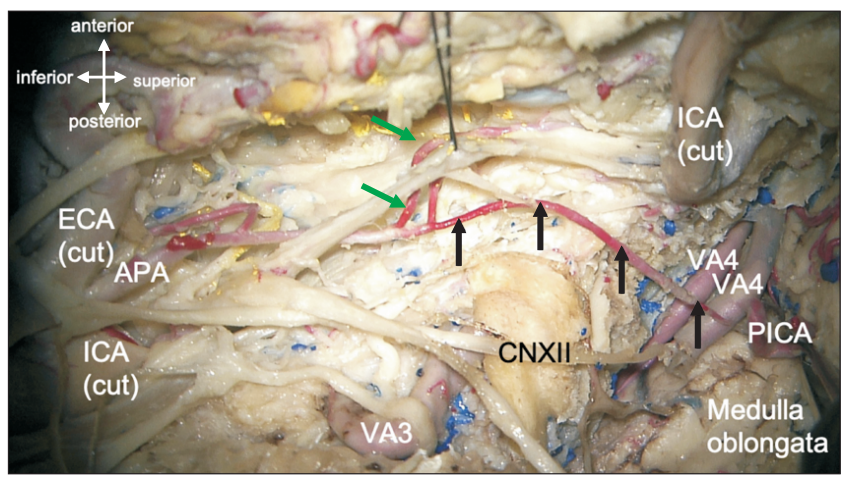

Fig. 2. Variant anastomosis i.e., hypoglossal artery (arrows) between the ascending pharyngeal artery (APA) and ipsilateral vertebral artery (VA4) via the hypoglossal canal, which has been dissected open and the hypoglossal nerve (CNXII) reflected inferiorly. Note the internal carotid artery (ICA) with its proximal part to the left and its distal part to the right and reflected up. Also note the external carotid artery (ECA) and third segment of the vertebral artery (VA3). The left vertebral artery where the anastomosis is seen is shown giving off the posterior inferior cerebellar artery (PICA). The sympathetic trunk is elevated with suture and the pharyngeal branches of the ascending pharyngeal artery are shown at the green arrows. lateral side.

\section{Discussion}

\section{Embryology}

During early embryonic stages (approximately weeks 6-8), the fetal carotid arteries supply much of the anterior and posterior cerebral circulation. The basilar artery is formed by the fusion of two paired neural arteries that course along the surface of the hindbrain $[1,2]$. The carotid arteries initially supply these neural arteries via trigeminal, hypoglossal, otic, and proatlantal intersegmental anastomoses $[1,2,4]$. When the posterior communicating arteries form from the fusion

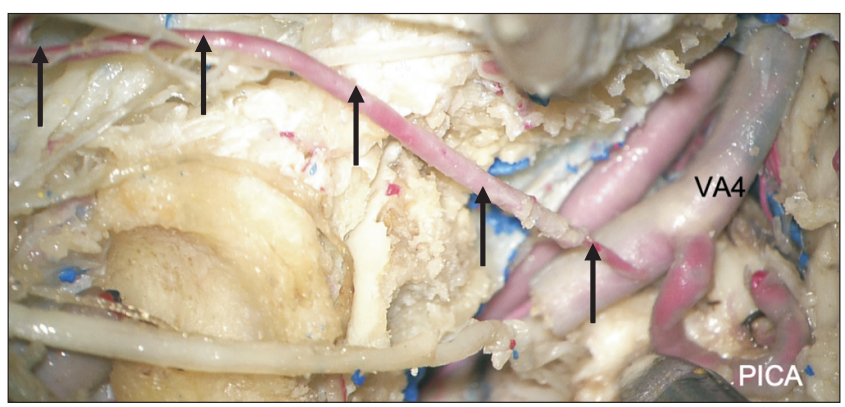

Fig. 3. Zoomed in photo of Fig. 2. Note the hypoglossal artery (arrows) traveling from the ascending pharyngeal artery and joining the ipsilateral and intracranial vertebral artery. The left posterior inferior cerebellar artery (PICA) is shown arising from the vertebral artery (VA4) segment of the vertebral artery near the joining of the hypoglossal artery.

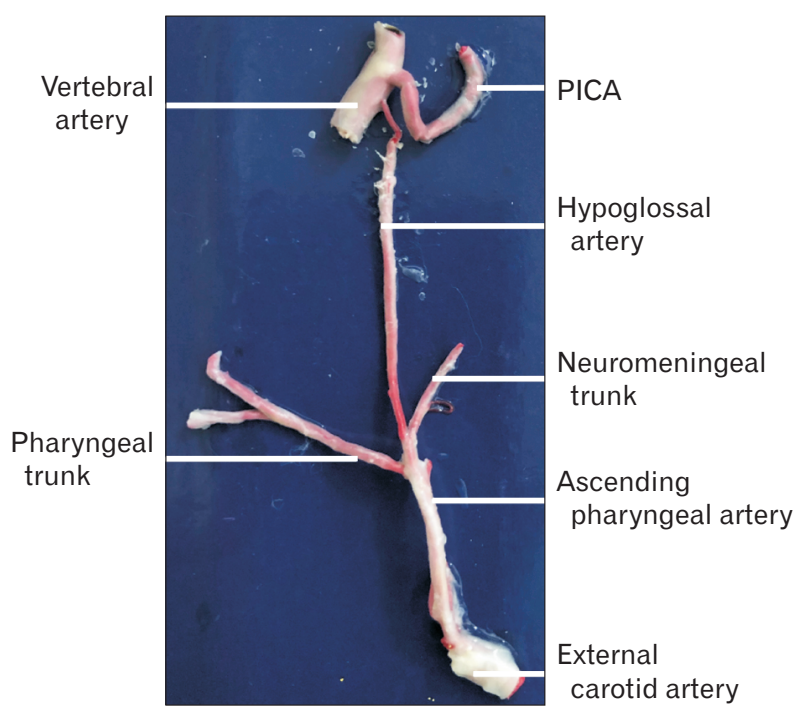

Fig. 4. The case reported herein following excision. PICA, posterior inferior cerebellar artery. 


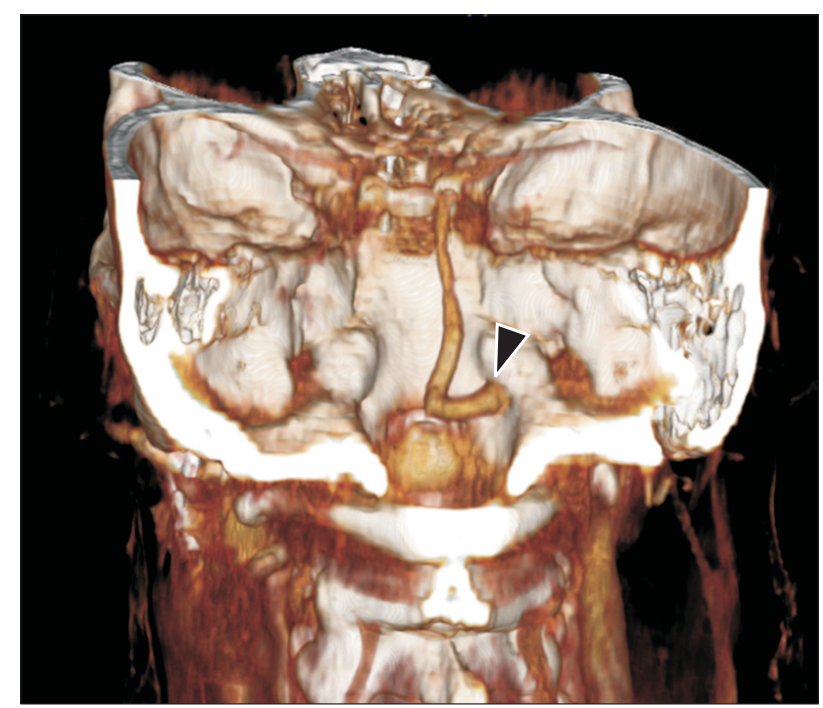

Fig. 5. Adult patient found to have a persistent hypoglossal artery (arrowhead) emerging from the hypoglossal canal on 3 dimensional reconstruction from a contrasted head and neck computed tomography. The vertical continuation of the vessel is the basilar artery.

between distal internal carotid and neural arteries, the four carotid-vertebrobasilar anastomoses regress and under normal circumstances the vertebral arteries become the major suppliers of the posterior cerebral circulation [2]. Failure of the primitive carotid-vertebrobasilar anastomoses to regress leads to persistent fetal anastomoses in adulthood.

\section{True persistent primitive hypoglossal artery}

A persistent fetal hypoglossal artery is the second most common anastomosis between the carotid and vertebrobasilar arterial systems (Figs. 5, 6) [1, 2, 4]. This variation is more common on the left side and in females [1]. The most frequent of these anastomoses originates from a persistent fetal trigeminal artery [4]. Less common anastomoses involve the otic and proatlantal arteries. If there is a persistent fetal hypoglossal artery, Brismar's diagnostic criteria suggest that an extracranial branch should originate from the internal carotid artery and anastomose with the vertebrobasilar trunk after passing through the hypoglossal canal [5]. The anastomosis typically originates between the $\mathrm{C} 1$ and $\mathrm{C} 2 \mathrm{lev}$ els of the internal carotid artery [1]. The branch then passes through a commonly enlarged hypoglossal canal and joins to an ipsilateral inferior segment of the basilar or vertebral artery. If this anastomosis is large, ipsilateral posterior communicating or vertebral arteries can be absent or hypoplastic. As mentioned previously, this communication is thought

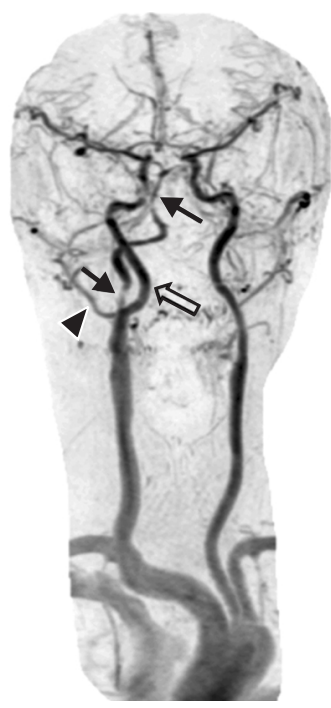

Fig. 6. Hypoglossal artery (black arrows) arises from the right internal carotid artery (outlined white arrow). The right external carotid artery is indicated by the black arrowhead.

to occur in $0.027 \%$ to $0.26 \%$ of the population $[2,4]$.

\section{Variant ascending pharyngeal-vertebral anastomosis}

In our case, we identified an external carotid-vertebrobasilar anastomosis (Figs. 2-4), which is rarer than the variation mentioned above that arises from the internal carotid artery $[3,4]$. To our knowledge only 10 cases have been reported previously and all have been identified using angiography [4]. Our cadaveric dissection (Figs. 2-4) showed the ascending pharyngeal branch of the external carotid artery giving off a hypoglossal branch that then coursed through the hypoglossal canal prior to joining the ipsilateral vertebral artery.

The ascending pharyngeal artery normally divides into two major trunks: an anterior pharyngeal trunk and a posterior neuromeningeal trunk. The latter trunk gives off jugular and hypoglossal branches with both supplying the dura mater of the posterior cranial fossa [6]. The hypoglossal branches also supply the hypoglossal nerve rootlets and contribute to the arterial arcade supplying the dens by joining the posterior ascending branch of the vertebral artery [6, 7].

Yamamoto et al. [4] and Uchino et al. [8] termed this persistent hypoglossal arterial variation a dilated ascending pharyngeal artery; they proposed that proximal fetal proatlantal intersegmental and distal fetal hypoglossal arteries contribute to this anastomosis. Lasjunias et al. [9] identified two types of proatlantal artery: type I and type II. A type I proatlantal intersegmental artery originates from the inter- 
nal carotid artery and represents a true persistent fetal proatlantal artery. A type II originates from the external carotid artery and shares a common trunk with the occipital artery. Uchino et al. [8] Okahara et al. [10] suggested that this variation represents a persistent first cervical intersegmental artery. In our case, and in the cases of Yamamoto et al. [4] and Uchino et al. [3] the proximal anastomoses originated from the ascending pharyngeal artery as opposed to the occipital artery, further defining this variant anastomosis.

Persistent carotid-vertebrobasilar anastomoses can be associated with atherosclerotic disease, intracranial ischemia, and intracranial aneurysms $[1-3,8]$. These variations should be identified prior to carotid endarterectomy (CEA), intravascular embolization, and other skull base surgeries as these persistent fetal vessels can serve as significant suppliers of the posterior cerebral circulation. In the presence of this variation, endarterectomy of external carotid during CEA is warranted. Some vascular surgeons believe that endarterectomy of external carotid artery may be neglected [11] without a significant consequence. However, neglecting endarterectomy of the external carotid artery is not suggested in the presence of a persistent external carotid-vertebrobasilar anastomosis. In addition, modification of technique during carotid artery stenting may be necessary in patients with this variation. These modifications include but are not limited to the selection of stent type (closed cell vs. open cell stents) and introduction of guidewires into the external carotid artery. Technical modifications in patients with persistent carotidvertebrobasilar anastomoses should be individualized based on pre-operative angiographic findings. Establishment of external carotid artery flow would be more important when vertebral artery hypoplasia is present on the contralateral side.

\section{ORCID}

Stephen J. Bordes: https://orcid.org/0000-0002-7507-0674

Sina Zarrintan: https://orcid.org/0000-0002-3968-0221

Joe Iwanaga: https://orcid.org/0000-0002-8502-7952

Marios Loukas: https://orcid.org/0000-0003-2811-6657

Aaron S. Dumont: https://orcid.org/0000-0002-8077-8992

R. Shane Tubbs: https://orcid.org/0000-0003-1317-1047

\section{Author Contributions}

Conceptualization: RST. Data acquisition: RST. Data analysis or interpretation: SJB, SZ, JI, ML, RST. Drafting of the manuscript: SJB. Critical revision of the manuscript: SZ, JI, ML, RST. Approval of the final version of the manuscript: all authors.

\section{Conflicts of Interest}

No potential conflict of interest relevant to this article was reported.

\section{Acknowledgements}

The authors sincerely thank those who donated their bodies to science so that anatomical research could be performed. Results from such research can potentially increase mankind's overall knowledge and thence improve patient care. Therefore, these donors and their families deserve our highest gratitude [12].

\section{References}

1. Srinivas MR, Vedaraju KS, Manjappa BH, Nagaraj BR. Persistent primitive hypoglossal artery (PPHA) - a rare anomaly with literature review. J Clin Diagn Res 2016;10:TD13-4.

2. Uchino A, Saito N, Okada Y, Kozawa E, Nishi N, Mizukoshi W, Inoue K, Nakajima R, Takahashi M. Persistent hypoglossal artery and its variants diagnosed by $\mathrm{CT}$ and MR angiography. Neuroradiology 2013;55:17-23.

3. Uchino A, Saito N, Kurita H. Anastomosis of the external carotid artery and the V3 segment of the vertebral artery (presumed persistent second cervical intersegmental artery) diagnosed by CT angiography. Surg Radiol Anat 2018;40:233-6.

4. Yamamoto R, Mori N, Nakae Y, Tanaka F, Johkura K. Anomalous anastomosis between the external carotid artery and vertebrobasilar artery via the hypoglossal canal: a case report and review of literature. Surg Radiol Anat 2019;41:849-52.

5. Brismar J. Persistent hypoglossal artery, diagnostic criteria. Report of a case. Acta Radiol Diagn (Stockh) 1976;17:160-6.

6. Hacein-Bey L, Daniels DL, Ulmer JL, Mark LP, Smith MM, Strottmann JM, Brown D, Meyer GA, Wackym PA. The ascending pharyngeal artery: branches, anastomoses, and clinical significance. AJNR Am J Neuroradiol 2002;23:1246-56.

7. Iwanaga J, Simonds E, Choi PJ, Oskouian RJ, Tubbs RS. Anatomical study of the posterior ascending artery: application to C2 pedicle screw placement. World Neurosurg 2018;112:e662-5.

8. Uchino A, Saito N. Persistent hypoglossal artery arising from the external carotid artery diagnosed by MR angiography. Surg Radiol Anat 2011;33:543-5.

9. Lasjaunias P, Théron J, Moret J. The occipital artery. Anatomynormal arteriographic aspects-embryological significance. 
Neuroradiology 1978;15:31-7.

10. Okahara M, Kiyosue H, Mori H, Tanoue S, Sainou M, Nagatomi $\mathrm{H}$. Anatomic variations of the cerebral arteries and their embryology: a pictorial review. Eur Radiol 2002;12:2548-61.

11. Ascher E, Hingorani A, Markevich N, Schutzer R, Yorkovich WR, Kallakuri S, Tsemekhim B. Carotid surgery without external carotid endarterectomy: a 6-year clinical experience with 1027 cases. Eur J Vasc Endovasc Surg 2003;25:458-61.
12. Iwanaga J, Singh V, Ohtsuka A, Hwang Y, Kim HJ, Moryś J, Ravi KS, Ribatti D, Trainor PA, Sañudo JR, Apaydin N, Şengül G, Albertine KH, Walocha JA, Loukas M, Duparc F, Paulsen F, Del Sol M, Adds P, Hegazy A, Tubbs RS. Acknowledging the use of human cadaveric tissues in research papers: recommendations from anatomical journal editors. Clin Anat 2021;34:24. 